\title{
MECHANISMS OF DELAYED LOWER URINARY TRACT STONE FORMATION
}

Fawad Arif and Alaiyi West

Mechanisms of Delayed Lower Urinary Tract Stone Formation after Holmium Laser Enucleation of Prostate: A Case Series and Review of Literature

Fawad Arif* and Alaiyi West

South Tees Hospitals NHS Foundation Trust, UK

*Corresponding author: Fawad Arif: drfawad@yahoo.com

Received: 27 December 2020; Accepted after revision: 13 January 2021; Published: 1 February 2021.

This article is distributed under the terms of the Creative Commons Attribution-NonCommercial 4.0 International License. CArif and West

\section{ABSTRACT}

\section{Materials and methods:}

We analysed Holmium Laser Enucleation of Prostate (HOLEP) database consisting of 1300 patients who presented more than 18 months after undergoing the procedure with lower urinary tract symptoms (LUTSvoiding lower urinary tract symptoms, dysuria, haematuria, recurrent urinary tract infections-UTIs) secondary to delayed lower urinary tract stone formation. Information was gathered from the case notes, imaging modalities, operation notes and pathology reports.

\section{Results:}

Three patients were identified who presented with delayed lower urinary tract stone formation 18 months after undergoing HOLEP.

Case 1: A 68-year-old presented with a 4-cm mobile bladder stone on a retained prostatic fragment 29 months after HOLEP.

Case 2: A 74-year-old presented with $2.5 \mathrm{~cm}$ bulbar urethral stone 18 months after HOLEP.

Case 3: A 77-year-old presented with dystrophic calcification of the entire prostatic fossa 60 months after HOLEP.

\section{Conclusion:}

Delayed lower urinary tract stone presentation is unusual after HOLEP. Recurrent urethral pain, recurrent UTI, gross haematuria and voiding lower urinary tract symptoms in the presence of a lower urinary tract stone (bladder, prostate and urethra) with a radiolucent centre on a background of HOLEP should raise the suspicion that this may represent calcification on a prostatic tissue fragment or dystrophic calcification of the residual prostate/prostatic fossa. Careful morcellation, inspection of the prostatic fossa on withdrawing the morcellator for large residual prostate fragments still attached to the prostatic bed/bladder neck or simply stuck to the fossa (usually in a clot) will reduce the risk of retainment of such a significant prostatic fragment that can potentially cause complications in the future. 


\section{INTRODUCTION}

Over the last decade, Holmium Laser Enucleation of Prostate (HOLEP) has emerged as a safe and effective transurethral treatment option worldwide for symptomatic benign prostatic enlargement (BPE) of any size. ${ }^{1,2}$ Its multifunctional nature, low complication rate, shorter hospital stay and cost effectiveness make it an attractive modality. ${ }^{3-5}$ There is paucity of data or information in the literature on incidence or factors leading to retention of prostatic tissue fragments following HOLEP. ${ }^{6}$ Most of these small fragments are passed spontaneously and do not require any intervention following the surgery. In rare occasions after the procedure, a significant fragment may necessitate surgical intervention. This is especially the case during initial few days to months, when the patient may present with persistence or recurrence of voiding symptoms, haematuria and urinary tract infections (UTIs).

\section{MATERIALS AND METHODS}

We analysed HOLEP database consisting of 1300 patients who presented more than 18 months after undergoing the procedure with lower urinary tract symptoms (LUTS) (voiding lower urinary tract symptoms, dysuria, haematuria and recurrent UTI) secondary to delayed lower urinary tract stone formation. Information was gathered from the case notes, imaging modalities, operation notes and pathology reports.

\section{RESULTS}

Three patients were identified who presented with delayed lower urinary tract stone formation 18 months after undergoing HOLEP.

\section{Case presentation 1 (bladder stone formation)}

A 68-year-old male with a background of noninsulin dependent diabetic, hypertensive, hypothyroidism and atrial fibrillation requiring Apixaban, underwent a straightforward, uneventful standard 3-lobe HOLEP for bothersome voiding symptoms, intermittent visible haematuria and chronic retention. Transrectal ultrasound of the prostate had confirmed a prostatic volume of $168 \mathrm{~mL}$ prior to the procedure. CT urogram had confirmed normal upper tracts. Cystoscopy was suggestive of a trilobar occlusive vascular prostate with contact bleeding and an unremarkable heavily trabeculated bladder with significant residual urine in keeping with known chronic retention (on an average $250-300 \mathrm{~mL}$ on previous ultrasound and post void bladder scans). The gentleman passed trial removal of catheter (TROC) the next morning and was discharged home.

On clinical review in 4 months' time, he was very satisfied with the outcome of the procedure. This was confirmed on uroflowmetry test when he had a maximum flow rate of $26 \mathrm{~mL}$ for a voided volume of $477 \mathrm{~mL}$ and a post void residual volume of $52 \mathrm{~mL}$. Although he did experience some transient stress incontinence for a few weeks after the procedure, the urinary tract was completely dry on the day. He had not been troubled with any further episodes of visible haematuria following the procedure. Histopathological analysis of the prostatic tissue confirmed $143 \mathrm{gm}$ of benign prostatic hyperplasia with no evidence of malignancy. He was discharged from the urology clinic.

The gentleman was referred back to the urology department with a 3-week history of frank, painless haematuria and a 4-month history of mixed, bothersome, lower urinary tract symptoms predominantly storage in nature including frequency and urgency 29 months after undergoing HOLEP. CT KUB (Figure 1) confirmed a $4-\mathrm{cm}$ bladder stone with a $2-\mathrm{cm}$ radiolucent centre suspicious for retained prostatic tissue fragment. Cystoscopy confirmed an open prostatic fossa in keeping with previous HOLEP and a 4-cm mobile bladder stone. A combination of Holmium LASER, Mauermayer stone punch and morcellation was utilised to fragment very hard stone and soft nucleus consisting of retained prostatic tissue fragment. The patient underwent a successful trial removal of the catheter the following morning. On further review 4 months later, there were no concerns. On histopathological analysis, not surprisingly there was no viable tissue in the specimen and it only confirmed 1 gm of extensively infarcted and necrotic tissue with foci of calcification.

\section{Case presentation 2 (urethral stone formation)}

A 74-year-old otherwise fit and well man underwent standard 3 lobe HOLEP for an 84-mL prostate causing high pressure urinary retention with acute kidney injury that normalised following indwelling catheterisation. There were no perioperative or postoperative concerns 
Figure 1. CT images of mobile bladder stone formation on retained prostatic fragment.

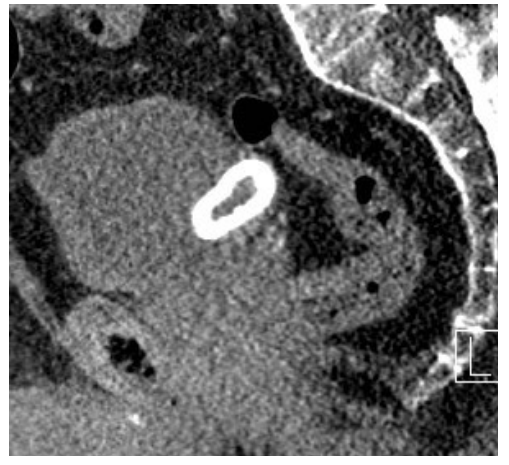

Sagittal

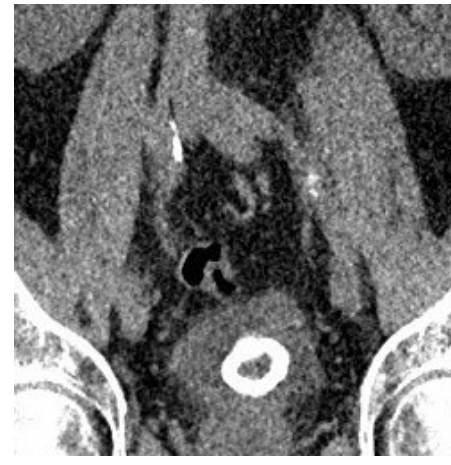

Coronal

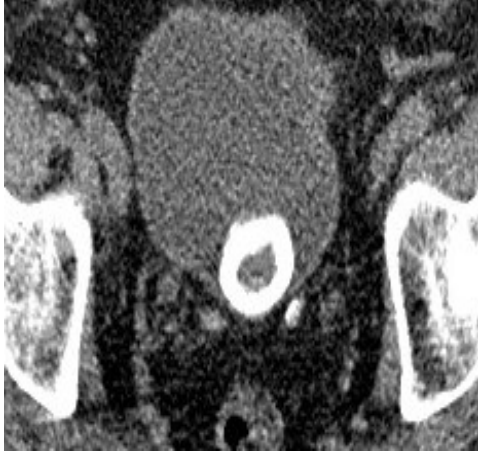

Transverse and he was discharged home the following morning after a successful trial removal of the catheter. A clinic review 4 months later was satisfactory when the gentleman had no concerns with the lower urinary tract, and was completely dry and histopathological analysis had confirmed 70 gm of benign tissue.

He was referred back to the urology clinic 18 months after the procedure with a 6-week history of poor flow and continuous dribbling incontinence for which he was utilising a body worn urinal attached to a leg bag (visible on subsequent $\mathrm{CT}$ images) $\mathrm{He}$ also presented with the sudden appearance of an obvious hard lump at the base of the penis over this period, which on subsequent flexible cystoscopy and CT imaging (Figure 2) was confirmed to be a $2.5 \mathrm{~cm}$ stone impacted in a stenosed bulbar urethra. He underwent a successful urethral dilatation, cystolitholapaxy and LASER fragmentation of the impacted urethral stone. Following the above procedure, cystoscopy otherwise confirmed an open bladder outlet and an unremarkable bladder. On a routine 4-month review, the patient was doing fine with no concerns.

Figure 2. CT images of urethral stone formation after HOLEP.

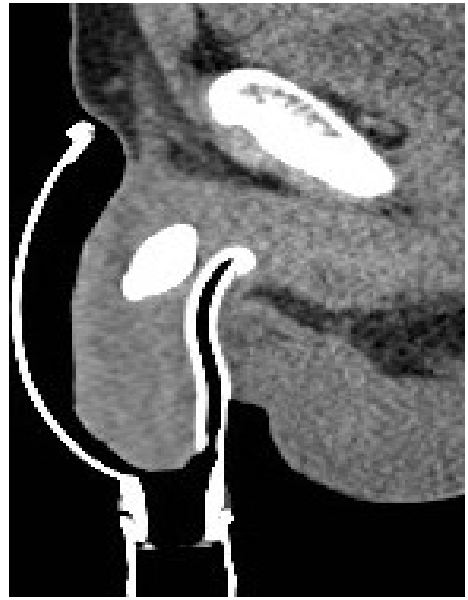

Sagittal

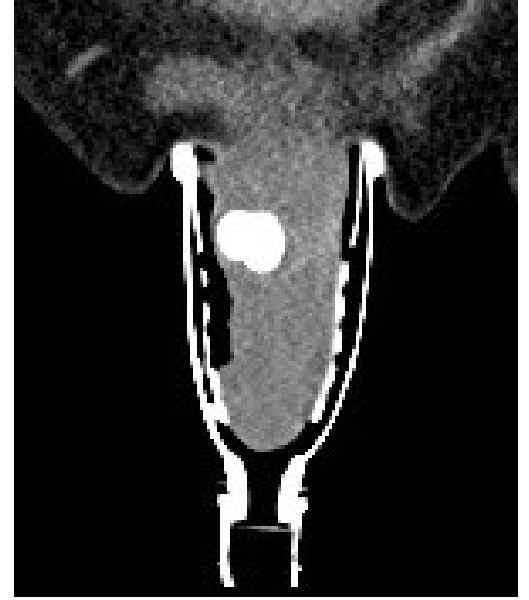

Coronal

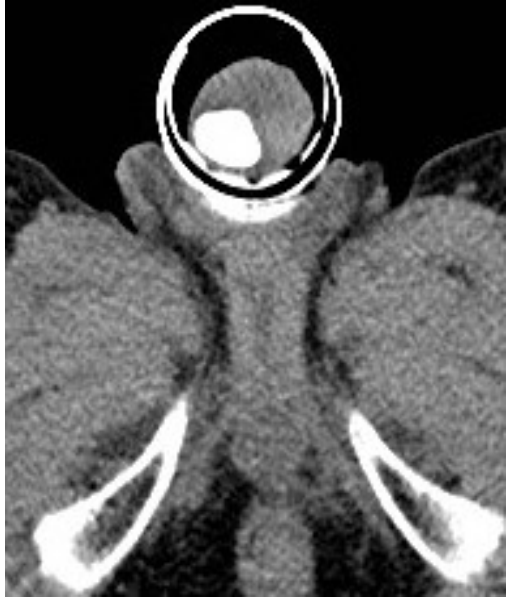

Transverse

J Endolum Endourol Vol 4(1):e17-e22; February 1, 2021.

This article is distributed under the terms of the Creative Commons Attribution-

Non Commercial 4.0 International License. (C) Arif and West 
Case presentation 3 (dystrophic prostatic calcification)

A 73-year-old gentleman with mild asthma and gout who had undergone HOLEP for bothersome voiding symptoms 5 years ago in the north west, while being investigated for visible haematuria and recurrent UTI, was found to have complete calcification of the entire prostatic fossa on cystoscopy and CT Urogram (Figure 3), which likely represented dystrophic calcification after HOLEP. He required further enucleation and morcellation of the prostatic cavity when $12 \mathrm{gm}$ of specimen showed extensive coagulative necrosis and dystrophic calcification in keeping with previous surgery.

\section{DISCUSSION}

HOLEP has been shown to be safe and effective modality in managing obstructive benign prostatic hypertrophy and it is comparable to transurethral resection of prostate and open prostatectomy. ${ }^{7-10}$ HOLEP has the advantage of greater prostate volume reduction and durable long-term results while maintaining low morbidity. ${ }^{11,12}$ Lower urinary tract stone formation after HOLEP is unusual and a rare complication.

According to our database encompassing more than 1300 procedures and reviewing the literature, there are two main causes for lower urinary tract stone formation following HOLEP, namely dystrophic calcification of the residual prostate/prostatic fossa and calcification/stone formation on retained prostatic fragment.

Dystrophic calcification is inappropriate mineralisation (calcification) in various tissues as a result of chronic inflammation, tissue damage and necrosis. Local deposits of calcium may occur in necrotic tissue such as old caseous tuberculous lesions, old infarcts and in fat necrosis associated with pancreatitis. Tissue undergoing slow degeneration as seen in arteries due to atheromatous degeneration, old thrombi or diseased/ abnormal heart valves can harbour calcium signifying dystrophic calcification. Necrosis or degeneration of tissue leads to release of enzymes causing breakdown of organic phosphates and alteration of $\mathrm{pH}$ facilitating calcium deposition.

In the urinary tract, dystrophic calcification has been noted in renal parenchymal disease, upper tract tumours and squamous cell cancer of the bladder due to schistosomiasis. ${ }^{13-15}$ Dystrophic calcification of the prostate and the residual prostatic bed, although rare, is a known entity and it has been previously reported in the literature after standard transurethral resection of the prostate, ${ }^{16} \mathrm{KTP}$ laser vaporisation of the prostate, ${ }^{17,18}$ cryotherapy ${ }^{19}$ and radiotherapy to the prostate was carried out especially in patients who had undergone a recent transurethral resection of the prostate. ${ }^{20}$

Lee et al. ${ }^{2}$ has reported de novo urethral stones/ calcification in the prostatic surgical bed after HOLEP.

Figure 3. CT images of dystrophic calcification following HOLEP.

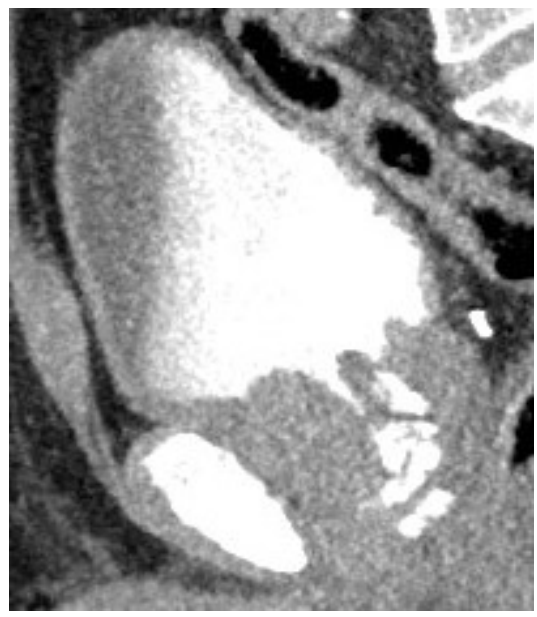

Sagittal

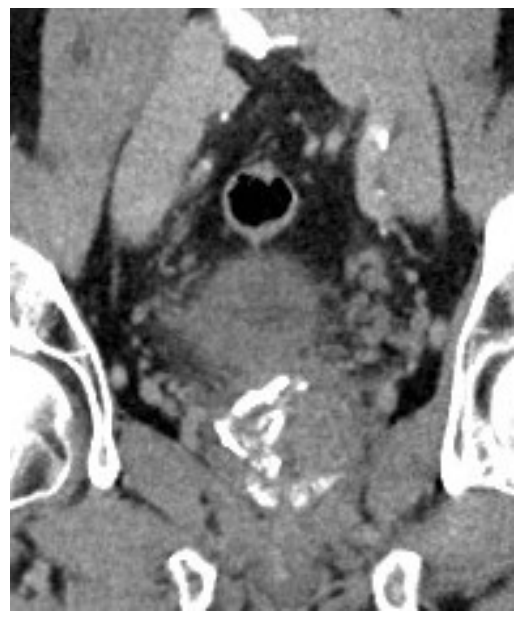

Coronal

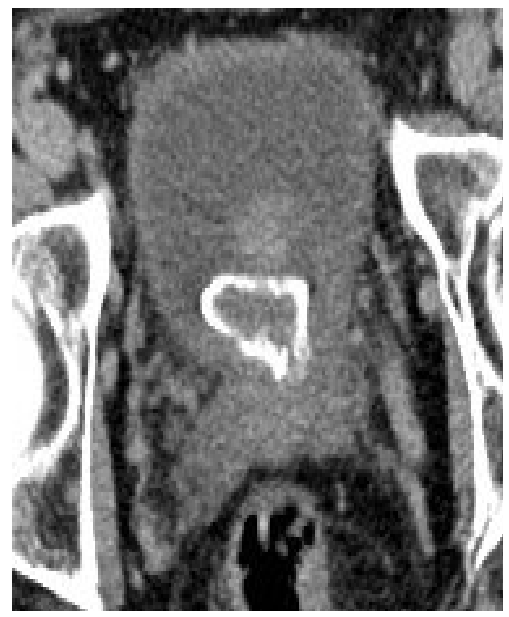

Transverse

J Endolum Endourol Vol 4(1):e17-e22; February 1, 2021.

This article is distributed under the terms of the Creative Commons Attribution-

Non Commercial 4.0 International License. (C) Arif and West 
In the study, 9 patients (of 877 patients in BPH database following HOLEP) were noted to have calculi attached to the surgical bed or bladder neck which were confirmed to be dystrophic calcification on histopathological analysis. Stasis of urine at prostatic fossa, debris attached to surgical bed of prostatic fossa and coagulative necrosis inducing dystrophic calcification are suggestive causes of stone formation after laser surgery.

As previously mentioned, retainment of small prostatic tissue fragments after HOLEP, which are passed spontaneously, is not an unusual occurrence especially with larger glands. In rare occasions, when it is more obvious in the initial postoperative period, surgical intervention may be necessary to remove a larger fragment. However, this is often by design especially in difficult cases when poor visibility and other patient factors may force the surgeon to postpone morcellation to another day. ${ }^{21}$

To our knowledge, there has been no cases reported in the literature of delayed presentation after HOLEP of bladder (29 months) and urethral stone (18 months) formation on (retained) prostatic tissue fragment.

In our case reports, the 4-cm bladder stone was completely mobile with a large radiolucent centre raising suspicion on CT KUB of this to likely represent calcification on a retained prostatic fragment that acted as a nidus for mineralisation. In the other case, the urethral stone impacted in the bulbar urethra had no attachments to the prostatic fossa. However, the narrow bulbar urethra that required dilatation during its removal could be a contributing factor leading to the impaction and enlargement of the stone at this site.

As these rather significant sized calcified prostatic tissue fragments presented years after HOLEP in the absence of significant symptoms in the interim such as voiding lower urinary tract symptoms, dysuria, haematuria, recurrent UTI and so on, it is plausible that the prostatic tissue sloughed/detached/broke off from the prostatic fossa/bladder neck attachment in later life and became calcified in due course.

\section{CONCLUSION}

Delayed lower urinary tract stone presentation is unusual after HOLEP. Recurrent urethral pain, recurrent UTI, gross haematuria and voiding lower urinary tract symptoms especially in the presence of a lower urinary tract stone (bladder, prostate and urethra) with a radiolucent centre on a background of HOLEP should raise the suspicion that this may represent calcification on a prostatic tissue fragment or dystrophic calcification of the residual prostate/prostatic fossa.

Careful morcellation and inspection of the prostatic fossa on withdrawing the morcellator for large residual prostate fragments still attached to the prostatic bed/ bladder neck or simply stuck to the fossa (usually in a clot) will reduce the risk of retainment of such a significant prostatic fragment that can potentially cause complications in the future.

\section{REFERENCES}

1. Tooher R, Sutherland P, Costello A, et al. A systemic review of holmium laser prostatectomy for benign prostatic hyperplasia. J Urol. 2004;171:1773. https:// doi.org/10.1097/01.ju.0000113494.03668.6d

2. Lee J, Oh J. Calculi in the prostatic surgical bed as a complication after holmium laser enucleation of the prostate. J Urol. 2018;15(5)238-41.

3. Elzayat EA, Habib E, Elhilali MM. Holmium laser enucleation of the prostate: A size-independent new "gold standard". Urology. 2005;66:108-13. https://doi. org/10.1016/j.urology.2005.06.006

4. Hettiarachchi JA, Samadi AA, Konno S, Das AK. Holmium laser enucleation for large (greater than 100 $\mathrm{mL}$ ) prostate glands. Int J Urol. 2002;9:233-6. https:// doi.org/10.1046/j.1442-2042.2002.00457.x

5. Suardi N, Gallina A, Salonia A, Briganti A, Deho F, et al. Holmium laser enucleation of the prostate and holmium laser ablation of the prostate: Indications and outcome. Curr Opin Urol. 2009;19:38-43. https://doi. org/10.1097/MOU.0b013e32831a7008

6. Sung HK, Changwon Y, Ninsoo C, et al. Factors affecting de novo urinary retention after holmium laser enucleation of prostate. PLoS One. 2014;9(1):e84938. https://doi.org/10.1371/journal.pone.0084938

7. Kuntz RM, Ahayi S, Lehrich K et al. Transurethral holmium laser enucleation of prostate versus transurethral electrocautery resection of the prostate: A randomised prospective trial in 200 patients. J Urol. 2004;172:1012. https://doi.org/10.1097/01.ju.0000136218.11998.9e

8. Ahyai SA, Lehrich K, Kuntz RM. Holmium laser enucleation versus transurethral resection of prostate: 3 -year followup results of a randomised clinical trial. 
Eur Urol. 2007;52:1456. https://doi.org/10.1016/j. eururo.2007.04.053

9. Elzayat EA, Elhilali MM. Holmium laser enucleation of prostate (HOLEP): The endourological alternative to open prostatectomy. Eur Urol. 2006;49:87. https:// doi.org/10.1016/j.eururo.2005.08.015

10. Kuntz RM, Lehrich K, Ahyai SA. Holmium laser enucleation of the prostate versus open prostatectomy for prostates greater than 100 grams: 5-year follow-up results of a randomised clinical trial. Eur Urol. 2008;53:160. https://doi.org/10.1016/j.eururo.2007.08.036

11. Gillin PJ, Aho TF, Frampton CM, et al. Holmium laser enucleation of the prostate: Results at 6 years. Eur Urol. 2008;53:744. https://doi.org/10.1016/j. eururo.2007.04.052

12. Elzayat EA, Elhilali MM. Holmium laser enucleation of the prostate (HOLEP); longterm results, reoperation rate and possible impact of learning curve. Eur Urol. 2007;52:1465. https://doi.org/10.1016/j. eururo.2007.04.074

13. Merchant SA, Amonkar PP. Genitourinary Bilharziasis: A review. Indian J Radiol Imaging. 2002;12(2):239-44.

14. Dyer RB, Chen MYM, Zagoria RJ. Abnormal calcifications in the urinary tract. Radiographics. 1998;18(6):1405-24. https://doi.org/10.1148/radiographics.18.6.9821191

15. Tsujimura A, Imazu T, Nishimura K, et al. Ureteropelvic junction obstruction with renal pelvic calcification: A case report. J Urol. 1993;150(6):1889-90. https://doi. org/10.1016/S0022-5347(17)35925-6
16. Valentin Z, Patrick B, Gaiter M, et al. Recurrent dystrophic calcification of the prostatic resection cavity after transurethral resection of the prostate: Clinical presentation and endoscopic management. J Endourol Case Rep. 2017;3(1):81-3. https://doi.org/10.1089/ cren.2017.0058

17. Jeon SW, Park YK, Chang SG. Dystrophic calcification and stone formation on the entire bladder neck after potassium-titanyl phosphate laser vaporization for the prostate: A case report. J Korean Med Sci. 2009;24:741-3. https://doi.org/10.3346/jkms.2009.24.4.741

18. Tasci AI, Tugcu V, Ozbay B, Mutlu B, Cicekler O. Stone formation in prostatic urethra after potassium-titanylphosphate laser ablation of the prostate for benign prostatic hyperplasia. J Endourol. 2009;23:1879-81. https://doi.org/10.1089/end.2008.0596

19. Dru C, Bender L. Dystrophic calcification of the prostate after cryotherapy. Case Rep Urol. 2014;2014:471385. https://doi.org/10.1155/2014/471385

20. Jones WA, Miller EV, Sullivan LD, et al. Severe prostatic calcification after radiation therapy for cancer. J Urol. 1979;121(6):828-30. https://doi.org/10.1016/ S0022-5347(17)57011-1

21. Gross AJ, Netsch C, Knipper S, Holzel J, Bach T. Complications and early postoperative outcome in 1080 patients after thulium vapoenucleation of the prostate: Results at a single institution. Eur Urol. 2013;63:859-67. https://doi.org/10.1016/j.eururo.2012.11.048 